\title{
On structural computations until fracture based on an anisotropic and unilateral damage theory
}

\author{
Martin Genet ${ }^{\mathrm{a}, 1, *}$, Lionel Marcin ${ }^{\mathrm{a}, 2}$, Pierre Ladevèze $^{\mathrm{a}, \mathrm{b}}$ \\ ${ }^{a}$ LMT-Cachan, ENS-Cachan/CNRS UMR8535/Paris VI University/UniverSud Paris \\ PRES, 61 avenue du Président Wilson, 94235 Cachan Cedex, France \\ ${ }^{b}$ EADS Foundation Chair "Advanced Computational Structural Mechanics"
}

\begin{abstract}
This paper describes the formulation and numerical implementation of a family of anisotropic and unilateral damage models for the prediction of damage and final rupture in engineering structures. The damage can be load-oriented, microstructure-oriented or (for the first time within this modeling framework) softening. The local equations are solved using a combination of fixed-point and Newton-Raphson algorithms, whose efficiencies are drastically improved through Aitken's relaxation and BFGS approximation. A delay-effect method is used to control the localization of damage, which leads to an objective calculation of the final rupture of structures.
\end{abstract}

Keywords:

Continuum damage mechanics; Anisotropic and unilateral damage;

Structural computation; Rupture; Delay-effect localization control

\footnotetext{
${ }^{*}$ Corresponding author. E-mail address: genet@lmt.ens-cachan.fr

${ }^{1}$ Current affiliation: Marie-Curie Fellow, Cardiac Biomechanics Laboratory, Department of Surgery, University of California at San Francisco, USA

${ }^{2}$ Current affiliation: Snecma Villaroche, France
} 


\section{Contents}

1 Introduction 3

2 The anisotropic and unilateral damage theory 6

2.1 State potential and state law . . . . . . . . . . 6

2.2 Thermodynamic forces . . . . . . . . . . . . . . . 9

2.3 Damage evolution laws . . . . . . . . . . . . . . . . . 10

2.3.1 The case of load-oriented damage . . . . . . . . . 10

2.3.2 The case of microstructure-oriented damage . . . . . . 12

2.3.3 The case of softening damage . . . . . . . . 16

3 Calculation of the local behavior $\quad 19$

3.1 The local loop . . . . . . . . . . . . . . . . . . . . . . . . . . 19

3.1.1 The fixed-point solver . . . . . . . . . . . . . 19

3.1.2 Acceleration of the solver . . . . . . . . . 20

3.1 .3 Performance ..................... 21

3.2 The behavior loop . . . . . . . . . . . . . . . . 22

3.2.1 The Newton-Raphson solver . . . . . . . . . . . . 24

3.2.2 Acceleration of the solver . . . . . . . . . 25

3.2 .3 Performance . . . . . . . . . . . . . 26

4 Control of the damage localization $\quad 26$

4.1 The delay-effect method . . . . . . . . . . . . . . . . 28

4.2 Illustrative examples . . . . . . . . . . . . . . . . . . 29

5 Conclusion $\quad 30$ 


\section{Introduction}

Since continuum damage mechanics (CDM) first appeared [Kachanov, 1958, 1999], it has been studied and improved by many research groups worldwide [Leckie, 1978; Ladevèze, 1983; Murakami, 1983; Lemaître, 1985; Simo and Ju, 1987; Chaboche, 1988], taught in many universities [Kachanov, 1986; Lemaître, 1992; Krajcinovic, 1996], and has become one of the classical tools of the structural mechanics community [Lemaître and Desmorat, 2005].

In practice, CDM consists in developing a model of the macroscopic behavior of a material in which microscopic damage is represented as a stiffness reduction. The form of the stiffness reduction is defined by the damage kinematics using damage variables, which are internal variables in thermodynamical terminology. The stiffness reduction, i.e. the magnitude of the damage variable, is defined as a function of the applied stress or strain using the damage kinetics. Damage models, and in particular their damage kinetics, usually involve several parameters (in addition to the classical Young and shear moduli and Poisson ratios) which must be identified experimentally through classical coupon tests. Then these models can be used to predict damage in engineering structures subjected to possibly complex loading and, for example, become part of a structural optimization process. Let us point out that homogenization techniques were recently used to derive damage kinematics and kinetics formally from an analysis on the damage scale, leading to generic models (i.e. models which are valid for a whole family of materials) for laminated composites [Ladevèze and Lubineau, 2002].

While early works concerned "only" unidirectional damage models, with applications to the creep rupture of metals [Kachanov, 1958, 1999; Leckie, 1978], CDM was rapidly extended to multidirectional models 
1 involving damage anisotropy [Cordebois and Sidoroff, 1982; Ladevèze, 2 1983; Murakami, 1983; Chaboche, 1984; Chow and Wang, 1987b], with 3 applications to the ductile [Lemaître, 1985; Chow and Wang, 1987a] and 4 fatigue [Chow and Wei, 1991] rupture of metals. More recently, CDM was 5 applied to laminated [Talreja, 1985, 1986; Voyiadjis and Kattan, 1993; 6 Ladevèze and Lubineau, 2002], woven [Lesne and Saanouni, 1993; Aubard, 7 1995; Ladevèze, 1995] and braided [Gorbatikh et al., 2007] composites, 8 to concrete [Peerlings et al., 1998; Pensée et al., 2002; Desmorat et al., 9 2007; Badel et al., 2007], etc. However, only a few published models are 10 capable of reproducing all aspects of cracks, including anisotropy and 11 unilaterality, especially when the orientation of damage is determined 12 by the loading, i.e. when the damage kinematics is not known a priori. 13 Indeed, in that case, anisotropy requires a tensor damage variable and the construction of a continuously differentiable potential involving tensors, 5 and tension/compression partitioning is not straightforward [Ladevèze, 1983; Chaboche, 1992; Carol and Willam, 1996; Desmorat, 2000; Ladevèze 17 and Letombe, 2000; Ladevèze, 2002]. This theoretical problem was solved 18 by Ladevèze within the anisotropic and unilateral damage theory, first 19 for second-order damage tensors [Ladevèze, 1983], then for more general 20 representations of damage [Ladevèze and Letombe, 2000; Ladevèze, 2002]. 21 Basically, the approach relies on a specific tension/compression partitioning 22 of stress or strain which takes into account the damage state in order 23 to ensure the continuity of the state law. Let us mention that another 24 solution to this problem for fourth-order damage tensors was proposed in 25 [Chaboche, 1995]. 
1 merical implementation of the family of models which belong to the sec-

2 ond version of the anisotropic and unilateral damage theory. This discussion follows the classical Newton-Raphson/Finite Element framework. Although other methods can be used to solve nonlinear partial differential equations over a domain [Ladevèze, 1999; Passieux et al., 2010], this is the most common method for dealing with material nonlinearities in science and engineering. All the developments presented here were carried out within Abaqus/Standard as $\mathrm{C}++$ UMats based on the software development platform of [Leclerc, 2010].

In the first part of the paper, we review the theory's basis and present several damage evolution laws which differ in the nature of the damage mechanisms they represent. Regarding the modeling, the first fundamental question concerning a crack network is whether its orientation is governed by the loading (e.g. the inter-yarn cracking of woven ceramic matrix composites (CMCs) [Ladevèze et al., 1994; Ladevèze, 1995; Lamon, 2001], the cracking of concrete [Desmorat et al., 2007], etc.) or by the microstructure (e.g. the intra-yarn cracking of woven CMCs [Ladevèze et al., 1994; Ladevèze, 1995; Lamon, 2001], the cracking of laminated composites [Talreja, 1985, 1986; Lafarie-Frenot et al., 2001; Ladevèze and Lubineau, 2002], etc.). The second question is whether the crack network becomes saturated or reaches a critical value beyond which it localizes to form a macroscopic crack [Needleman, 1988; Pijaudier-Cabot and Benallal, 1993; Peerlings et al., 1998; Ladevèze et al., 2000]. Examples are presented for each situation. It is worth mentioning that the softening case is addressed in this modeling framework for the first time.

In the second part, we discuss the resolution of the local behavior at the integration point level. We use a fixed-point algorithm to solve the 
1 local equations; we also present and evaluate several relaxation schemes, 2 including Aitken's. We also focus on the inversion of the state law. As will 3 be seen later on, this law is nonlinear even if all the internal variables are 4 fixed, and it requires a specific solver. Therefore, we use a Newton-Raphson 5 algorithm; we also present and evaluate several optimization techniques, 6 including BFGS operator updating and Aitken's relaxation.

7 In the third part, we discuss the control of damage localization be8 yond the critical point of a softening model. We use a delay-effect method 9 [Ladevèze et al., 2000] to overcome the loss of ellipticity [Pijaudier-Cabot and

10 Benallal, 1993; Peerlings et al., 1998] and control the localization of damage 11 in the form of a macroscopic crack, which eliminates any pathological mesh 12 dependency. Again, let us mention that the simulation of localization in the 13 context of this damage framework is presented here for the first time.

14

We end up with a relatively complete, efficient and robust computational environment for anisotropic and unilateral damage within the popular Abaqus/Standard finite element code.

\section{The anisotropic and unilateral damage theory}

\subsection{State potential and state law}

State potential. The damage framework introduced in [Ladevèze and Letombe, 2000; Ladevèze, 2002] is based on the following general form of the potential of elastic energy:

$$
2 \rho \phi(\underline{\underline{\sigma}}, \underline{\underline{\underline{ }}}, \underline{\underline{\underline{ }}})=\langle\underline{\underline{\sigma}}\rangle_{+}^{S}: \underline{\underline{\underline{\underline{S}}}}:\langle\underline{\underline{\sigma}}\rangle_{+}^{S}+\langle\underline{\underline{\sigma}}\rangle_{-}^{S_{0}}: \underline{\underline{\underline{\underline{S_{0}}}}}:\langle\underline{\underline{\sigma}}\rangle_{-}^{S_{0}}+\underline{\underline{\sigma}}: \underline{\underline{\underline{Z}}}: \underline{\underline{\sigma}}
$$

where $\underline{\underline{S_{0}}}$ is the compliance tensor of the undamaged material, which is always active in compression; $\underline{\underline{\underline{S}}}$ is a damaged compliance, which is active 
1 only in tension (initially, $\underline{\underline{\underline{S}}}(t=0)=\underline{\underline{\underline{\underline{S_{0}}}}}$; and $\underline{\underline{\underline{\underline{Z}}}}$ is an additional compliance, 2 which is active both in tension and in compression (initially, $\underline{\underline{\underline{Z}}}(t=0)=0$ ).

3 Let us first observe that this potential gives maximum freedom in terms 4 of damage modeling, which enables one to deal with load-oriented damage, 5 i.e. damage whose direction is not known a priori: the damage kinematics 6 is not set a priori, but is defined completely by the damage evolution laws. 7 Actually, there are no damage variables associated with specific damage 8 mechanisms; the damage variables of the model are the whole compliance

tensors $\underline{\underline{\underline{S}}}$ and $\underline{\underline{\underline{\underline{Z}}}}$. Thus, any compliance can be reached from the initial compliance, which makes the model equivalent to an eighth-order damage tensor model [Lemaître et al., 2009].

Let us mention that we also considered strain-based formulations, but we had to abandon that idea because it could lead to cases in which the damaged stiffness tensor ceases to be positive definite before the actual stiffness in the loading direction gets to zero, which would make it impossible to model final rupture.

In order to deal with the crack closure effect, the model distinguishes clearly the tension state from the compression state: the stress tensor is divided into a positive part and a negative part, each associated with a different compliance operator. To ensure the continuous differentiability of the state potential, i.e. the continuity of the state law, this partitioning is carried out in a specific way which takes into account the middle operator. It is wellknown that the coupling between tensorial damage and tension/compression partitioning is not straightforward [Ladevèze, 1983; Chaboche, 1992]. There- 
1 fore, we use the following definitions of positive and negative stresses:

$$
\left\{\begin{array}{l}
\langle\underline{\underline{\sigma}}\rangle_{+}^{S}=\underline{\underline{\underline{S}}}^{-1 / 2}:\left\langle\underline{\underline{\underline{S}}}^{1 / 2}: \underline{\underline{\sigma}}\right\rangle_{+} \\
\langle\underline{\underline{\sigma}}\rangle_{-}^{S_{0}}={\underline{\underline{\underline{\underline{S_{0}}}}}}^{-1 / 2}:\left\langle{\underline{\underline{\underline{\underline{S_{0}}}}}}^{1 / 2}: \underline{\underline{\sigma}}\right\rangle_{-}
\end{array}\right.
$$

2 where \langle\rangle$_{+/-}$denotes the positive/negative decomposition of a second-

3 order symmetric tensor (obtained by taking the positive/negative eigen-

4 values alone). It is important to note that unless both the positive part

5 and the negative part are defined that way the state law is discontinuous.

6 With these definitions, the continuous differentiability of the potential can

7 be clearly shown by introducing Equation (2) into Equation (1):

$2 \rho \phi(\underline{\underline{\sigma}}, \underline{\underline{\underline{S}}}, \underline{\underline{\underline{\underline{Z}}}})=\left\langle\underline{\underline{\underline{\underline{S}}}}^{1 / 2}: \underline{\underline{\sigma}}\right\rangle_{+}:\left\langle\underline{\underline{\underline{\underline{S}}}}^{1 / 2}: \underline{\underline{\sigma}}\right\rangle_{+}+\left\langle{\underline{\underline{\underline{\underline{S_{0}}}}}}^{1 / 2}: \underline{\underline{\sigma}}\right\rangle_{-}:\left\langle{\underline{\underline{\underline{\underline{S_{0}}}}}}^{1 / 2}: \underline{\underline{\sigma}}\right\rangle_{-}+\underline{\underline{\sigma}}: \underline{\underline{\underline{\underline{Z}}}}: \underline{\underline{\sigma}}$

8 whose continuous differentiable property is trivial [Ladevèze and Letombe,

9 2000; Desmorat, 2000]. As a direct consequence of this property, the state

10 law (which is the first derivative of the potential) will always be contin-

11 uous, and the compliance operator (the second derivative) will always be

12 symmetric.

13 Finally, our framework also enables us to distinguish between damage which is highly dependent on the tension/compression state (i.e. tension damage, added to $\underline{\underline{\underline{S}}}$ ) and damage which is independent of the tension/compression state (i.e. shear damage, added to $\underline{\underline{\underline{\underline{Z}}}}$ ).

17 State law. The state law, derived from the elastic energy potential, is simply:

$$
\underline{\underline{\epsilon}}=\frac{\partial \rho \phi}{\partial \underline{\underline{\sigma}}}=\underline{\underline{\underline{S}}}:\langle\underline{\underline{\sigma}}\rangle_{+}^{S}+\underline{\underline{\underline{\underline{S_{0}}}}}:\langle\underline{\underline{\sigma}}\rangle_{-}^{S_{0}}+\underline{\underline{\underline{\underline{Z}}}}: \underline{\underline{\sigma}}
$$


1 2.2. Thermodynamic forces

$2 \quad$ First of all, let us define the following two thermodynamic forces, which

3 are linked directly to the energy release rates associated with the evolutions

4 of the damage variables:

$$
\left\{\begin{array}{l}
\underline{\underline{\underline{\underline{Y_{S}}}}}=2 \frac{\partial \rho \phi}{\partial \underline{\underline{\underline{S}}}}=\langle\underline{\underline{\sigma}}\rangle_{+}^{S} \otimes\langle\underline{\underline{\sigma}}\rangle_{+}^{S} \\
\underline{\underline{\underline{\underline{Y_{Z}}}}}=2 \frac{\partial \rho \underline{\underline{\rho}}}{\partial \underline{\underline{\underline{Z}}}}=\underline{\underline{\sigma}} \otimes \underline{\underline{\sigma}}
\end{array}\right.
$$

5 These thermodynamic forces or the corresponding damage variables cannot

6 be associated with specific damage mechanisms and, therefore, cannot be 7 used as such to drive all damage mechanisms; several additional thermody8 namic forces must be defined in order to drive any type of damage.

For example, we will see that while tension damage can be driven correctly by $\underline{\underline{\underline{\underline{Y_{S}}}}}$ (which is highly load-oriented, see Equation (5)) or its projections onto specific directions, this is not the case for shear damage. Because of the positive part of $\underline{\underline{\sigma}}$, only the tension part (i.e. the positive eigenvalue) drives the evolution of damage. Besides, since $\underline{\underline{Y}}_{Z}$ is active also in compression, we will not use it to drive any type of damage. Therefore, we must define another thermodynamic force to drive shear damage (see Sections 2.3.1 and 2.3.2). This additional force is simply a rotation of $\underline{\underline{\underline{\underline{Y_{S}}}}}$ defined as:

$$
\begin{gathered}
\underline{\underline{\underline{\underline{Y_{S^{\prime}}}}}}=\left(\underline{\underline{R_{\pi / 2}}}\langle\underline{\underline{\underline{\sigma}}}\rangle_{+}^{S}\right)_{\mathrm{sym}} \otimes\left(\underline{\underline{R_{\pi / 2}}}\langle\underline{\underline{\sigma}}\rangle_{+}^{S}\right)_{\mathrm{sym}} \\
\text { with } \underline{\underline{\underline{R_{\pi / 2}}}}=\left[\begin{array}{rr}
0 & -1 \\
1 & 0
\end{array}\right]
\end{gathered}
$$

Since all the previous thermodynamic forces are proportional to stresses, 10 they could not be used to model the localization of damage (i.e. the soften- 
1 ing phenomenon). Therefore, we will need to define an additional thermo-

2 dynamic force which is proportional to strains (see Section 2.3.3):

$$
\underline{\underline{\underline{\underline{X_{S}}}}}=\underline{\underline{\underline{S}}} \underline{\underline{\underline{\underline{Y_{S}}}}} \underline{\underline{\underline{S}}}
$$

3 2.3. Damage evolution laws

4 Several damage evolution laws can be defined depending on the very 5 nature of the damage they represent: is this damage load-oriented or 6 microstructure-oriented? Does it reach saturation or does it localize into 7 a macroscopic crack? We present examples for each situation, including 8 (for the first time) localizing damage. The proposed formulation is closely 9 modeled after the associated classical framework with normality rule and 10 isotropic hardening [Lemaître et al., 2009].

11 2.3.1. The case of load-oriented damage

12 For the sake of simplicity, from here on, we will use essentially the clas-

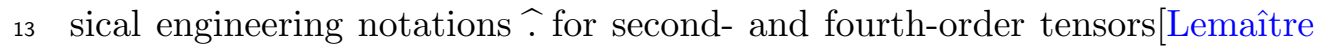
14 et al., 2009].

15 In the case of load-oriented damage, one can use the load-oriented ther16 modynamic forces directly to drive the evolution of damage. Thus, we define 17 the following effective thermodynamic force and its maximum over time:

$$
\left\{\begin{array}{l}
z=\left(a \operatorname{Tr}\left(\underline{\underline{\widehat{Y}_{S}}}\right)^{n+1}+(1-a) \operatorname{Tr}\left({\underline{\underline{\widehat{Y}_{S}}}}^{n+1}\right)\right)^{1 / n+1} \\
\bar{z}(t)=\sup _{\tau \leq t} z(\tau)
\end{array}\right.
$$

18 Then the corresponding evolution of the damage variables is:

$$
\left\{\begin{array}{l}
\dot{\hat{S}}=\dot{\alpha} \frac{\partial z}{\partial \widehat{\widehat{Y_{S}}}}=\dot{\alpha} \frac{a \operatorname{Tr}\left(\underline{\underline{Y_{S}}}\right)^{n} \underline{\underline{1}}+(1-a) \underline{\underline{\widehat{Y}_{S}}}}{\bar{z}^{n}} \\
\underline{\underline{\hat{Z}}}=\dot{b} \frac{b \frac{\underline{\bar{Y}_{S^{\prime}}}}{\overline{z^{n}}}}{}
\end{array}\right.
$$


1 where $\alpha$ is the "hardening variable", a function of $\bar{z}$ which must be cali-

2 brated experimentally. Usually, one assumes a shape depending on several

3 parameters which must be identified. For example, the following shape was

4 used in [Ladevèze and Letombe, 2000; Ladevèze, 2002; Genet et al., 2012]:

$$
\alpha=k\left\langle\frac{\sqrt{\bar{z}}-\sigma_{0}}{\sigma_{0}}\right\rangle_{+}^{2}
$$

5 where $\sigma_{0}$ characterizes the onset of cracking and $k$ its intensity. (In the case

6 of a unidirectional load, $\sqrt{\bar{z}}$ is simply the magnitude of the stress.) The

7 corresponding responses of this and other laws are compared in Figure 3 of

8 Section 2.3.3.

9 The dissipation associated with this evolution law is:

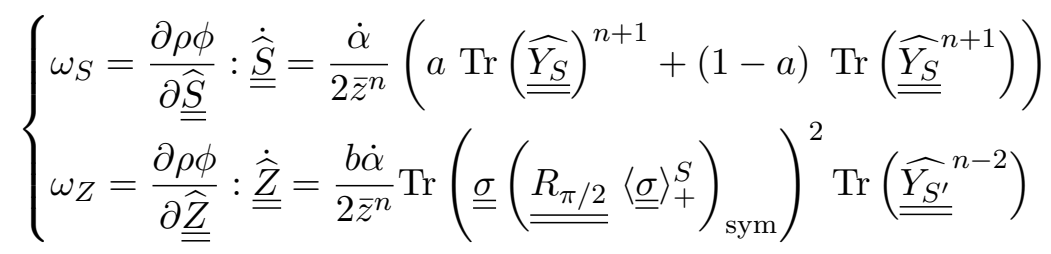

10 which is trivialy positive.

As can be seen in Equation (9), the evolution of damage can be tuned using parameters $a, b$ and $n$ and can be made highly load-oriented. For example, if $a=1$, the tension damage is fully isotropic; but if $a=0$, the larger $n$, the more anisotropic the tension damage. Regarding shear damage, its magnitude is directly proportional to $b$. To illustrate the influence of $a$ 
and $b$, let us define the relative angular tension and compression moduli:

$$
\left\{\begin{array}{l}
\tilde{E}_{t}(\theta)=\frac{\underline{\underline{N}}(\theta): \underline{\underline{\underline{\underline{S_{0}}}}: \underline{\underline{N}}(\theta)}}{\underline{\underline{\underline{N}}(\theta):(\underline{\underline{\underline{S}}}+\underline{\underline{\underline{Z}}}): \underline{\underline{\underline{N}}}(\theta)}} \\
\tilde{E}_{c}(\theta)=\frac{\underline{\underline{N}}(\theta): \underline{\underline{\underline{\underline{\underline{S_{0}}}}}: \underline{\underline{N}}(\theta)}}{\underline{\underline{N}}(\theta):\left(\underline{\left.\underline{\underline{\underline{S_{0}}}}+\underline{\underline{\underline{\underline{Z}}}}\right): \underline{\underline{N}}(\theta)}\right.}
\end{array}\right.
$$

with $\underline{\underline{N}}(\theta)=\underline{n}(\theta)^{t} \underline{n}(\theta)$

$$
\text { and } \underline{\underline{n}}(\theta)=\left[\begin{array}{c}
\cos \theta \\
\sin \theta
\end{array}\right]
$$

1 These relative angular tension and compression moduli corresponding to

2 a given amount of damage in different directions for an initially isotropic

3 material are presented in Figure 1 for several values of $a$ and $b$. (In all

4 cases, $n=2$; due to symmetry, only one quadrant was required for each set

5 of parameters.) One can see that a rotation of the load leads simply to a

6 rotation of the damage morphology.

\section{2.3.2. The case of microstructure-oriented damage}

8 In this case, the load-oriented thermodynamic forces cannot be used

9 directly, but must be projected onto the a priori known damage directions.

10 For example, for a crack network which is orthogonal to $\underline{n}$ and parallel to $\underline{t}$, 11 we define the projectors:

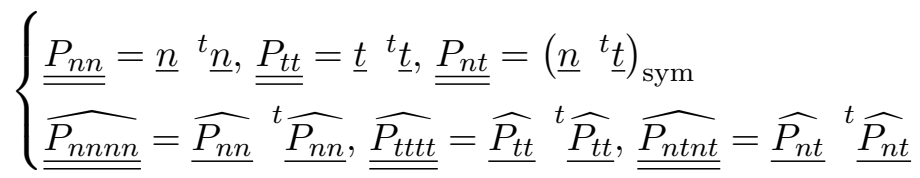

12 and the effective thermodynamic force:

$$
\left\{\begin{array}{l}
z=\left(a \operatorname{Tr}\left(\underline{\underline{\widehat{Y_{S}}}}\right)^{n+1}+(1-a) \operatorname{Tr}\left(\underline{\underline{\widehat{P_{n n n n}}}} \underline{\underline{\widehat{Y}_{S}}} \underline{\underline{P_{n n n n}}}\right)\right)^{1 / n+1} \\
\bar{z}(t)=\sup _{\tau \leq t} z(\tau)
\end{array}\right.
$$




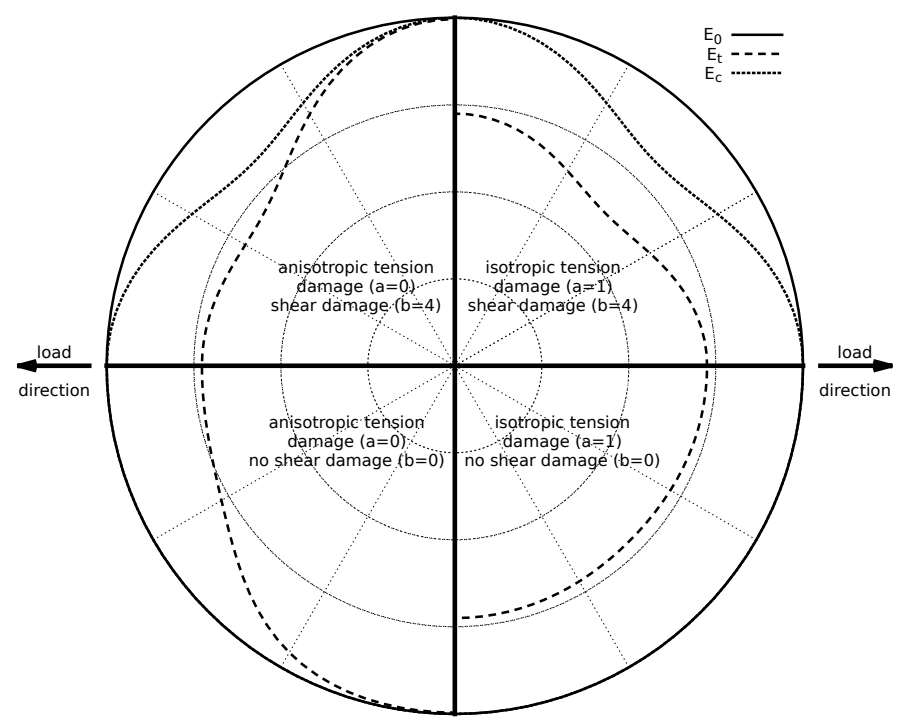

(a) First load direction

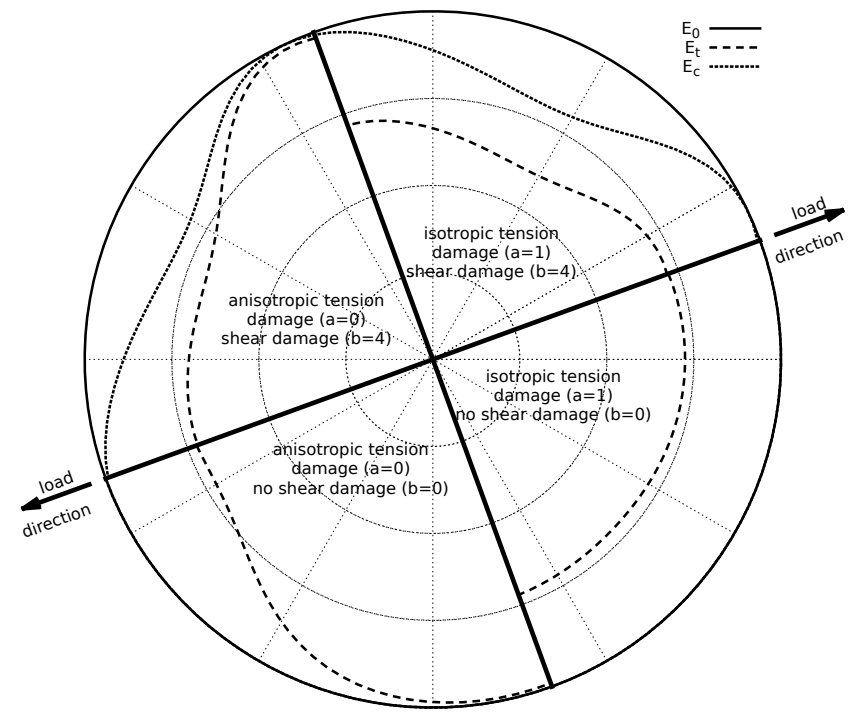

(b) Second load direction

Figure 1: Damaged angular tension and compression moduli using a load-oriented damage evolution law and two load directions for several values of $a$ and $b$ (top: shear damage activated, $b=4$; bottom: no shear damage, $b=0$; left: anisotropic tension damage, $a=0$; right: isotropic tension damage, $a=1$ ) 
1 The corresponding evolution of the damage variables is:

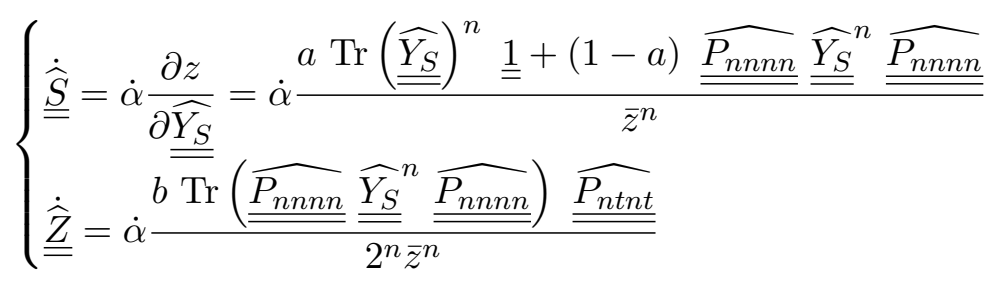

2 where $\alpha$ is again the "hardening variable" to be calibrated experimentally 3 (see Section 2.3.1).

4 Let us note that in the case of a unidirectional tension load normal to the 5 crack this evolution law is almost the same as the load-oriented law defined 6 in Section 2.3.1. The only small difference is due to the positive stress, 7 which is slightly different from the complete stress even for a unidirectional 8 tension load and, therefore, slightly modifies the effect of projections. This 9 difference is not significant, as can be seen in Figure 3 of Section 2.3.3 where the evolution laws are compared.

11 In this case, the dissipation is:

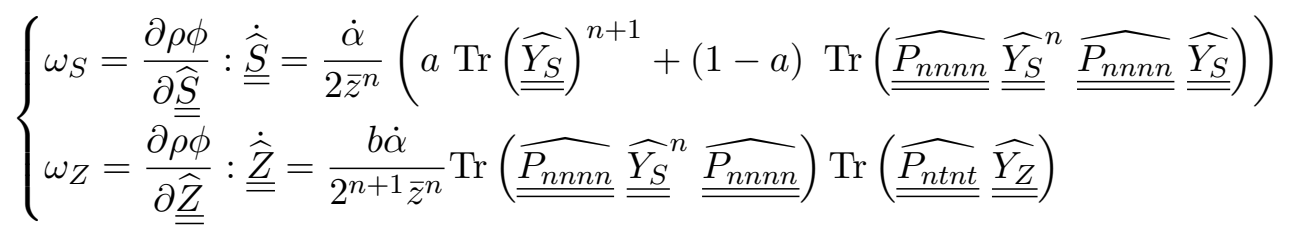

12 which is also positive.

13 Equation (15) clearly shows that in this case the evolution of damage is 14 oriented by the microstructure. Relative angular tension and compression 15 moduli corresponding to a given amount of damage in different directions 16 are presented in Figure 2 for several values of $a$ and $b$ (in all cases, $n=2$ ).

17 One can see that in this case a change in the load direction modifies the 18 amount of damage, but not its morphology. 


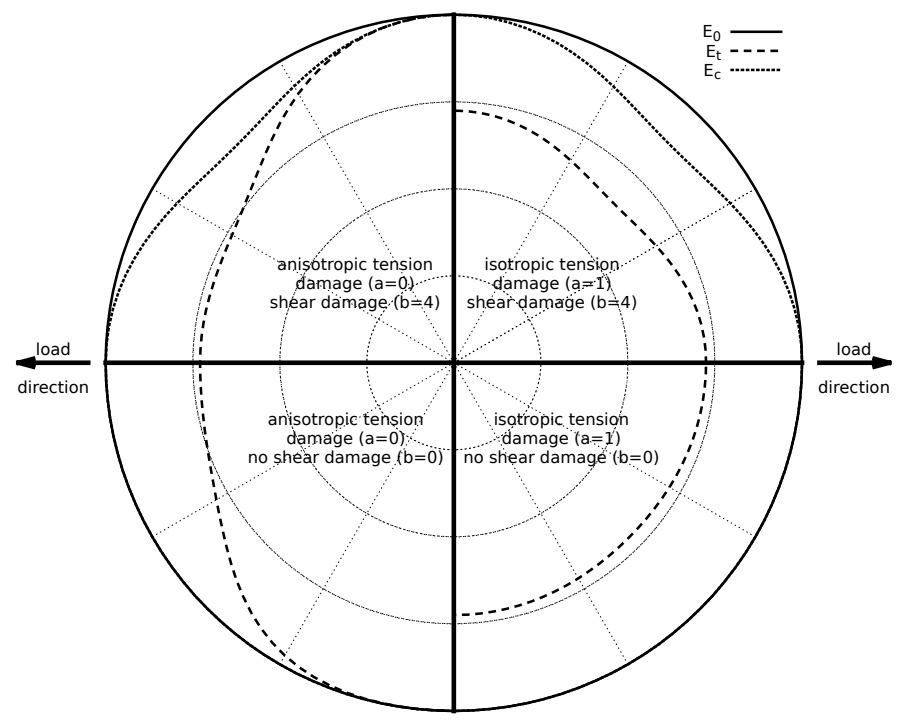

(a) Load direction perpendicular to the crack

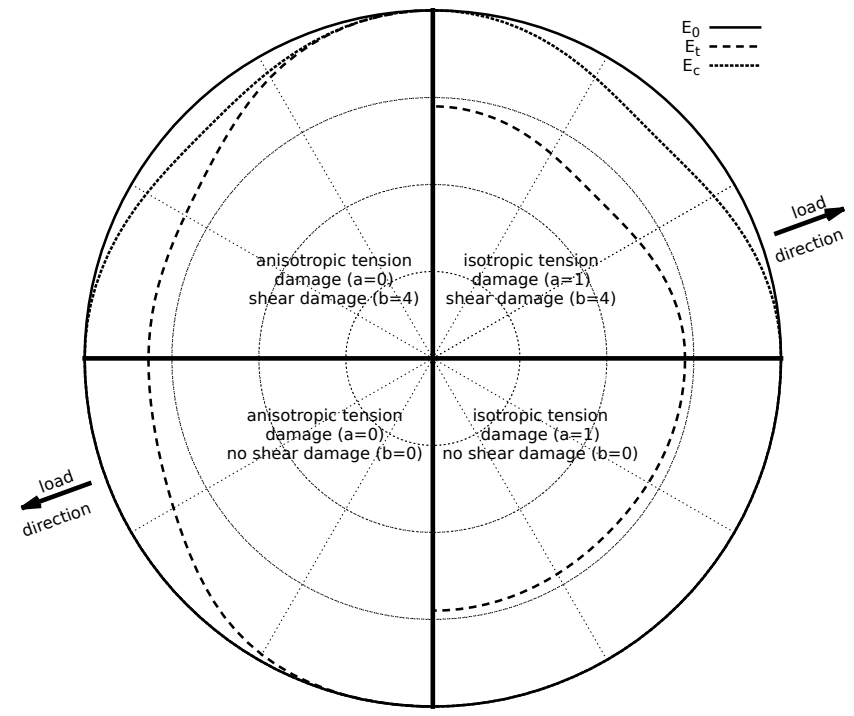

(b) Load direction at $20^{\circ}$ of the normal to the crack

Figure 2: Damaged angular tension and compression moduli using a microstructureoriented damage evolution law and two load directions for several values of $a$ and $b$ (top: shear damage activated, $b=4$; bottom: no shear damage, $b=0$; left: anisotropic tension damage, $a=0$; right: isotropic tension damage, $a=1$ ) 
1 2.3.3. The case of softening damage

2 This is the first presentation of a softening damage evolution law in the 3 proposed anisotropic and unilateral damage theory. In this case, formula4 tions similar to those presented in Section 2.3.1 for load-oriented damage 5 and in Section 2.3.2 for microstructure-oriented damage can be applied, but

6 thermodynamic forces proportional to strains rather than stresses must be 7 used (see Section 2.2). Indeed, beyond the critical point, the stress decreases 8 and, thus, a thermodynamic force dependent on the stress alone could not 9 cause the damage to increase. Therefore, one must consider a force based 10 either on the effective stress or on the strain. We chose the latter. Thus, for 11 a load-oriented mechanism, we use the effective thermodynamic force:

$$
\left\{\begin{array}{l}
z=\left(a \operatorname{Tr}\left(\underline{\underline{\widehat{X}_{S}}}\right)^{n+1}+(1-a) \operatorname{Tr}\left({\underline{\underline{\widehat{X}_{S}}}}^{n+1}\right)\right)^{1 / n+1} \\
\bar{z}(t)=\sup _{\tau \leq t} z(\tau)
\end{array}\right.
$$

12 with the evolution of the damage variable:

$$
\underline{\underline{\hat{S}}}=\dot{\alpha} \frac{\partial z}{\partial \underline{\underline{\widehat{X_{S}}}}}=\dot{\alpha} \frac{a \operatorname{Tr}\left(\underline{\underline{\widehat{X_{S}}}}\right)^{n} \underline{\underline{1}}+(1-a) \underline{\underline{{\widehat{X_{S}}}^{n}}}}{\bar{z}^{n}}
$$

13 For a microstructure-oriented mechanism, we use the effective thermody14 namic force:

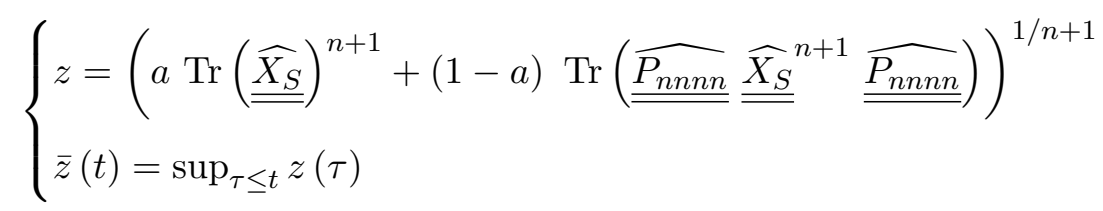

15 with the evolution of the damage variable:

$$
\underline{\underline{\hat{S}}}=\dot{\alpha} \frac{\partial z}{\partial \underline{\underline{\widehat{X_{S}}}}}=\dot{\alpha} \frac{a \operatorname{Tr}\left(\underline{\underline{\widehat{X_{S}}}}\right)^{n} \underline{\underline{1}}+(1-a) \underline{\underline{\underline{P_{n n n n}}}}}{\bar{z}^{n}} \underline{\underline{{\widehat{X_{S}}}^{n}}} \underline{\underline{\underline{P_{n n n n}}}}
$$


$1 \alpha$ is still the "hardening variable" to be calibrated experimentally (see Sec-

2 tion 2.3.1), but it must be redefined because the effective thermodynamic

3 forces are now proportional to the strains. For example, one can use the

4 following shape:

$$
\alpha=\left\{\begin{array}{ccc}
0 & \text { if } & \sqrt{\bar{z}} \leq \epsilon_{0} \\
k\left(\frac{\sqrt{\bar{z}}-\epsilon_{0}}{\epsilon_{1}-\sqrt{\bar{z}}}\right)^{2} & \text { if } & \epsilon_{0} \leq \sqrt{\bar{z}} \leq \epsilon_{1} \\
+\infty & \text { if } & \sqrt{\bar{z}} \geq \epsilon_{1}
\end{array}\right.
$$

5 where $\epsilon_{0}$ characterizes the onset of cracking, $\epsilon_{1}$ the final rupture, and $k$ the

6 amount of damage. (In the case of a unidirectional load, $\sqrt{\bar{z}}$ is simply the

7 longitudinal strain.) The responses of this and previous laws are compared

8 in Figure 3.

$9 \quad$ In this case, the dissipation is:

$\omega=\frac{\partial \rho \phi}{\partial \underline{\underline{\widehat{S}}}}: \underline{\underline{\hat{S}}}=\frac{\dot{\alpha}}{2 \bar{z}^{n}}\left(a \operatorname{Tr}\left(\underline{\underline{\widehat{X_{S}}}}\right)^{n} \operatorname{Tr}\left(\underline{\underline{\widehat{Y_{S}}}}\right)+(1-a) \operatorname{Tr}\left(\underline{\underline{\widehat{P_{n n n n}}}} \underline{\underline{\widehat{X}_{S}^{n}}} \underline{\underline{\underline{P_{n n n n}}}} \underline{\underline{Y_{S}}}\right)\right)$

which is also positive.

Let us mention that only tension damage was considered here because the introduction of shear damage would lead to the same problem as strainbased formulations (see the remark in Section 2.1): sometimes the damaged stiffness tensor could cease to be positive definite before the actual stiffness in the loading direction gets to zero.

Another element must be added to this law in order to control the end of the damaging process. Since tension damage alone is being considered, the only internal variable is the tensor $\underline{\underline{\underline{S}}}$ itself. (The case of shear damage with a softening law, which involves the second internal variable $\underline{\underline{\underline{Z}}}$, has not yet been addressed.) Thus, the full damage criterion can be based directly 
1 on the eigenvalues of $\underline{\underline{S}}$ :

$$
\exists i \quad / \quad \frac{1}{M E_{0}} \leq \lambda_{\underline{\underline{S}}}^{i} \leq \frac{M}{E_{0}} \quad \Longrightarrow \quad \text { rupture }
$$

2 where $\lambda_{\underline{\underline{S}}}^{i}$ are the eigenvalues of $\underline{\underline{\widehat{S}}}$ and $M$ is a large number (in practice, we 3 use $\left.M=10^{3}\right)$. When this limit is reached, the corresponding integration

4 point is considered to be broken and the damage ceases to evolve. It is worth 5 mentioning that this criterion can be applied at no substantial additional 6 cost because $\underline{\underline{\widehat{S}}}$ must be diagonalized anyway in order to calculate $\sqrt{\underline{\underline{\widehat{S}}}}$.

7 The responses of the model under unidirectional tension-compression

8 loading using the damage evolution laws presented in Sections 2.3.1, 2.3.2

9 and 2.3.3 are compared in Figure 3. (In the case of the microstructure10 oriented law, the load was orthogonal to the cracks.) One can see that 11 every model recovers its stiffness in compression, that the load-oriented and 12 microstructure-oriented non-softening models are nonseparable, and that 13 the law presented in this section does have a softening character.

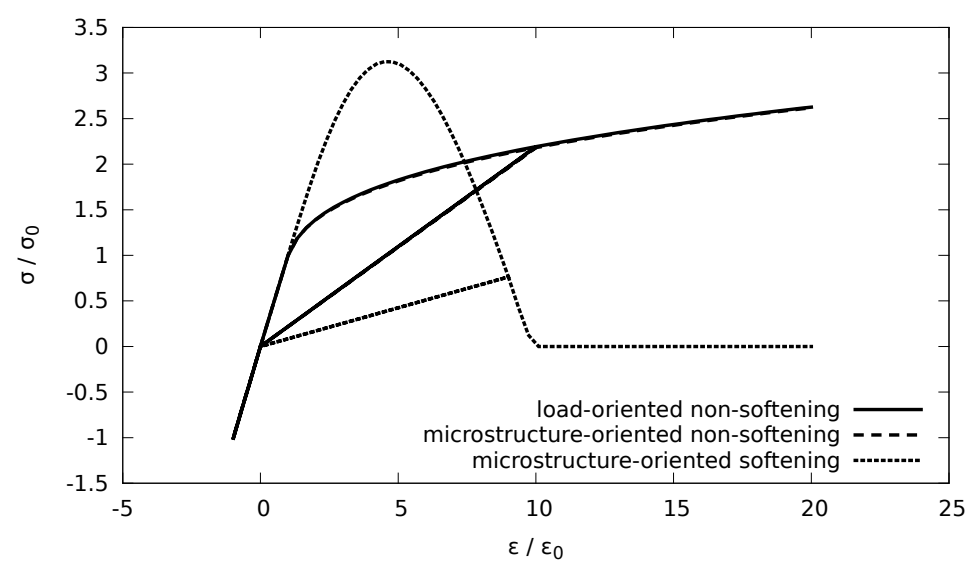

Figure 3: The responses of the model under unidirectional tension-compression loading $\left(k=10^{-5}, \epsilon_{1} / \epsilon_{0}=10\right)$ using different damage evolution laws 


\section{3. Calculation of the local behavior}

2 Let us now present the key aspects of the numerical implementation of 3 the family of models presented in Section 2 based on the anisotropic and 4 unidirectional damage theory. In this section, only non-softening damage

5 evolution laws are considered. The softening case will be discussed in Section 64.

7 3.1. The local loop

$8 \quad$ The local loop is run for each integration point and at each global itera9 tion of each load increment. The input is $\underline{\underline{\epsilon}}^{l, i}$, the total strain tensor at load 10 increment $l$ and global iteration $i$, and the output consists of the damage 11 tensors $\underline{\underline{\underline{S}}}^{l, i}$ and $\underline{\underline{\underline{Z}}}^{l, i}$, the corresponding damage "hardening" variable $\alpha^{l, i}$, 12 and the stress tensor $\underline{\underline{\sigma}}^{l, i}$. Starting here, in order to simplify the notation, 13 the subscripts $l, i$ will be omitted.

14

15

\subsubsection{The fixed-point solver}

This set of nonlinear equations could be solved using a Newton-Raphson method, but the very different units and magnitudes of the unknowns would lead to very unbalanced problems. Besides, most of the derivatives in the equations are difficult to calculate or even to approximate. For these reasons, 
1 we chose to use the following fixed-point algorithm instead:

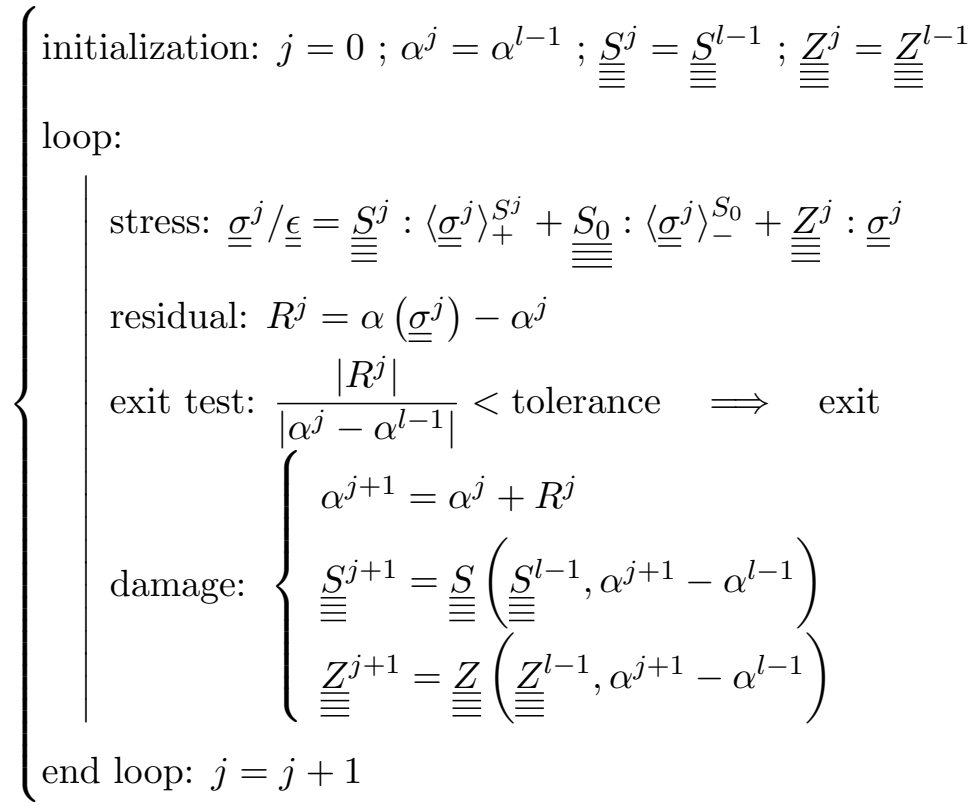

2 where $\alpha$ is the function defined in Equations (10) or (21), and $\underline{\underline{\underline{S}}}$ and $\underline{\underline{\underline{Z}}}$ are 3 the functions defined in Equations (9) or (15). Concerning the tolerance,

4 in the absence of a reference value for the magnitude of the residual of the

5 proposed algorithm, we used a stagnation criterion with a tolerance of $10^{-3}$.

6 3.1.2. Acceleration of the solver

7 The fixed-point method can be viewed as a Newton-Raphson method 8 with a unit search direction and, therefore, can oscillate greatly. This is 9 particularly true in our case because of the presence of unilateral conditions 10 (damage can only increase, see Equations (8) and (14)) and because in 11 practice Algorithm (24) has very poor convergence or no convergence at all.

12 Therefore, we propose two relaxation methods which improve the algorithm 13 convergence drastically. The only modification to Algorithm (24) concerns 
1 the damage increase line, which becomes:

$$
\alpha^{j+1}=\alpha^{j}+s^{j} R^{j}
$$

2 where $s^{j}$ is defined as follows:

3 Basic relaxation. One can consider that relaxation is required only if con-

4 vergence fails to occur after a given number of iterations. This leads to the

5 following very simple relaxation scheme:

$$
s^{j}=\frac{1}{1+E(j / N)}
$$

6 where $E$ is the classical integer part operator and $N$ is the predefined number 7 of iterations.

8 Aitken's relaxation. Another acceleration scheme considered here is Aitken's 9 relaxation, whose excellent performance was demonstrated in recent works 10 [Kassiotis et al., 2010]. This can be viewed as a search direction optimization 11 based on previous iterations. Then, $s^{j}$ is defined as:

$$
s^{j}= \begin{cases}1 & \text { if } j=0 \\ -s^{j-1} \frac{R^{j-1}}{R^{j}-R^{j-1}} & \text { if } j>0\end{cases}
$$

12 3.1.3. Performance

13 The performance of each of the options proposed for the resolution of 14 the model's equations was evaluated using a very simple problem involving 15 no structural effect: a single linear quadrangular element with four integra16 tion points was subjected to pure unidirectional tension through symmetry 17 conditions and prescribed displacements (see Figure 4). The damage law 18 considered was that of Section 2.3.1, which leads to the most difficult local 19 loop because damage is stress-driven (see Figure 3). Figure 5 shows how the 
1 methods compare in terms of the number of iterations and time. Clearly,

2 Aitken's relaxation was found to be much more efficient than fixed-value 3 relaxation, and it will be used from now on. For example, Figure 5(b) shows 4 a $75 \%$ gain compared to the case where relaxation occurs after 10 uncon5 verged iterations. Figure 5(c) also shows that this acceleration reduces the 6 cost of the local loop to about $10 \%$ of that of the global loop, which is a 7 very satisfactory ratio.

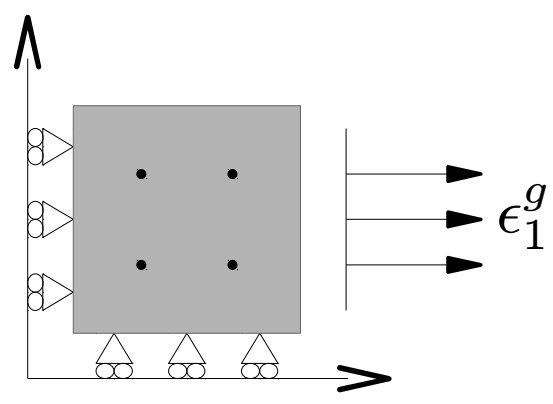

Figure 4: The problem used for the evaluation of the performance of the solver $\left(\epsilon_{1}^{g}\right.$ denotes the applied strain)

\subsection{The behavior loop}

9 In the previous discussion of the local loop (see Section 3.1), we never 10 explained how to calculate $\underline{\underline{\sigma}}$ when all the internal variables are fixed (see 11 the second line of Algorithm (24)). While this step is straightforward for 12 most existing models, it is not for ours. Because of the partitioning of $\underline{\underline{\sigma}}$ 13 into positive and negative parts, Equation (4) is nonlinear even with fixed 14 operators, so the problem can be formulated as follows:

$$
\left\{\begin{array}{l}
\text { with } \underline{\underline{\epsilon}}, \underline{\underline{\underline{\underline{S}}}}, \underline{\underline{\underline{\underline{S_{0}}}}} \text { and } \underline{\underline{\underline{\underline{Z}}} \text { known, find } \underline{\underline{\sigma}} \text { such that }} \\
\underline{\underline{\epsilon}}=\underline{\underline{\underline{S}}}:\langle\underline{\underline{\sigma}}\rangle_{+}^{S}+\underline{\underline{\underline{\underline{S_{0}}}}}:\langle\underline{\underline{\sigma}}\rangle_{-}^{S_{0}}+\underline{\underline{\underline{\underline{Z}}}}: \underline{\underline{\sigma}}
\end{array}\right.
$$




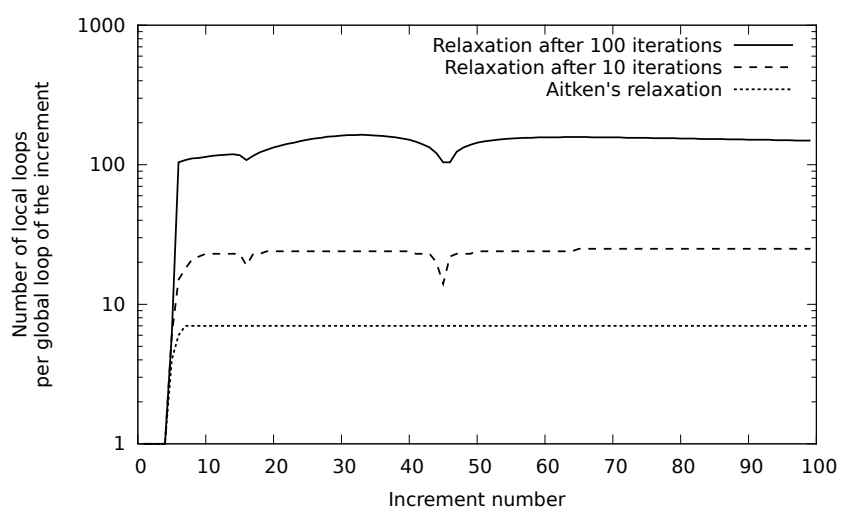

(a) iterations

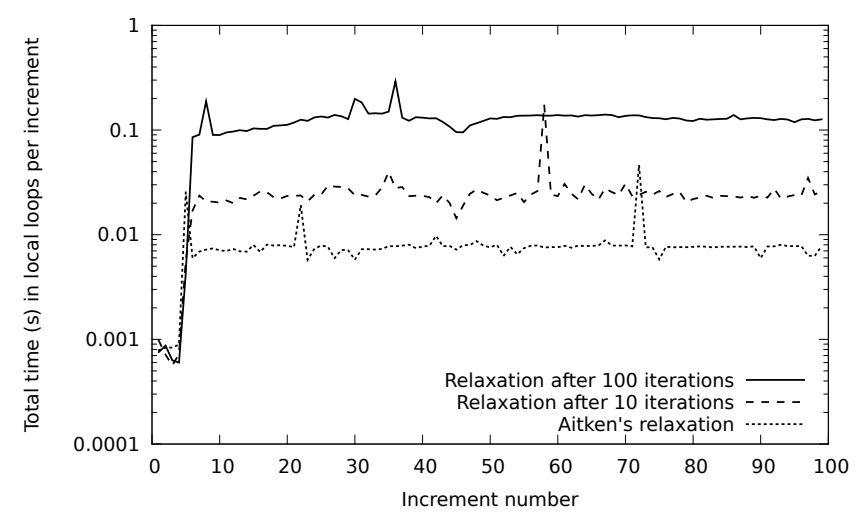

(b) time

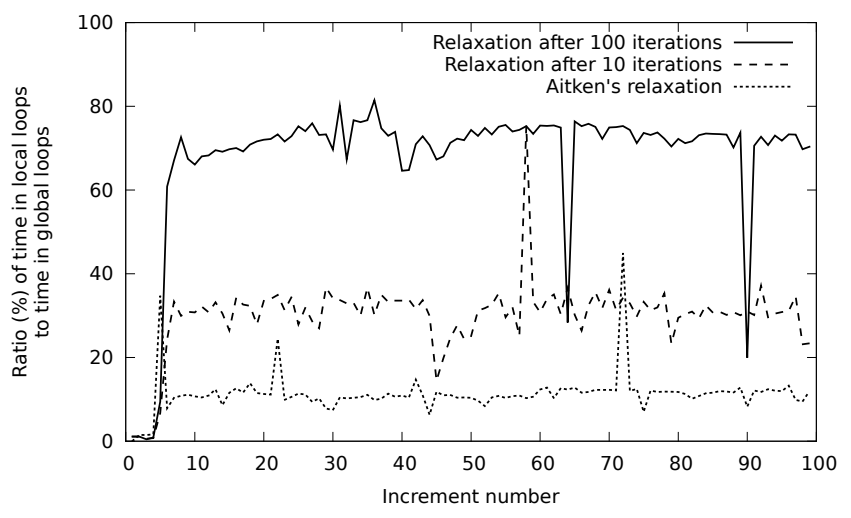

(c) time ratio

Figure 5: Performances of the local loop acceleration methods 


$$
\left\{\begin{array}{l}
\text { initialization: } k=0 ; \underline{\underline{\sigma}}^{k}=\underline{\underline{\sigma}}^{l-1} \\
\text { loop: } \\
\mid \begin{array}{l}
\text { residual: } \underline{\underline{R}}^{k}=\underline{\underline{\epsilon}}-\underline{\underline{\underline{S}}}:\left\langle\underline{\underline{\sigma}}^{k}\right\rangle_{+}^{S}-\underline{\underline{\underline{\underline{S_{0}}}}}:\left\langle\underline{\underline{\sigma}}^{k}\right\rangle_{-}^{S_{0}}-\underline{\underline{\underline{Z}}}: \underline{\underline{\sigma}}^{k} \\
\text { exit test: }\left\|\underline{\underline{R}}^{k}\right\|<\text { tolerance } \Longrightarrow \text { exit } \\
\text { stress: } \underline{\underline{\sigma}}^{k+1}=\underline{\underline{\sigma}}^{k}+\underline{\underline{\underline{D}}}^{k}: \underline{\underline{R}}^{k} \\
\text { end loop: } k=k+1
\end{array}
\end{array}\right.
$$

3 for which several search directions $\underline{\underline{\underline{D}}}^{k}$ can be used. The actual tangent

4 direction is not an option because one cannot derive the state law (4) with

5 respect to $\underline{\underline{\sigma}}$ in the general case where $\langle\underline{\underline{\sigma}}\rangle_{+}^{S} \neq 0$ and $\langle\underline{\underline{\sigma}}\rangle_{-}^{S_{0}} \neq 0$. One can

6 choose, for example, the initial operator $\underline{\underline{\underline{\underline{H_{0}}}}}=\underline{\underline{\underline{\underline{S_{0}}}}}{ }^{-1}$, which is not a good

7 direction, especially when the damage is significant, but which is fast because

8 the calculation of the operator is very inexpensive. Another option is to use

9 the quasi-secant operator, defined as:

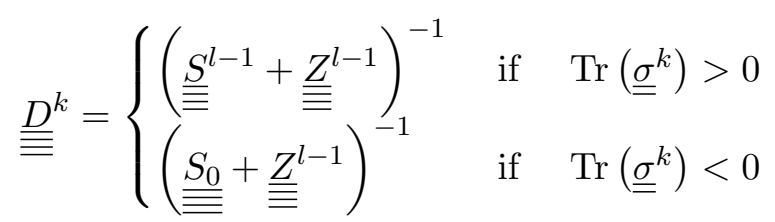

10 which is a much better search direction, but also a more expensive one 11 because these operators are usually not saved and must be recalculated at 12 each iteration. Other means of drastically improving the performance of the 13 solver will be presented in Section 3.2.2.

14 Regarding tolerance, in practice, since the residual of the proposed al15 gorithm is a strain, we use $10^{-9}$. 
1 3.2.2. Acceleration of the solver

2 In practice, the initial operator converges very slowly, or even does not 3 converge at all if the damage is significant. The secant operator defined 4 in Equation (30) generally converges very poorly, too. Therefore, we pro5 pose two acceleration methods in order to improve the convergence of the 6 algorithm drastically.

7 BFGS search direction. The first acceleration scheme one can consider is 8 the BFGS method [Matthies and Strang, 1979], whose ability to provide a 9 very good compromise between cost and quality for the search direction is well-known: a quasi-tangent direction is generated at the cost of $4 k$ additional scalar products compared to the initial direction [Matthies and Strang, 1979]. The algorithm for the calculation of $\underline{\underline{\underline{D}}}^{k}$ is well-known and will not 13 be recalled here; the only specificity is that the secant operator defined in

Equation (30) is used for the central operator because the tangent operator cannot be derived formally.

Aitken's relaxation. Another acceleration considered here is Aitken's relaxation, which was already used in Section 3.1.2 and which can also be viewed as a search direction optimization based on previous iterations. The only modification to Algorithm (29) is the last line, which becomes:

$$
\begin{aligned}
& \underline{\underline{\sigma}}^{k+1}=\underline{\underline{\sigma}}^{k}+s^{k} \underline{\underline{\underline{D}}}^{k}: \underline{\underline{R}}^{k} \\
& \text { with } \quad s^{k}= \begin{cases}1 & \text { if } k=0 \\
-s^{k-1} \frac{\underline{\underline{R}}}{\left(\underline{\underline{R}}^{k-1}-\underline{\underline{R}}^{k-1}\right):\left(\underline{\underline{R}}^{k}-\underline{\underline{R}}^{k}-\underline{\underline{R}}^{k-1}\right)} & \text { if } k>0\end{cases}
\end{aligned}
$$

16 For the same reason as for the BFGS acceleration, we use the secant operator 17 defined in Equation (30) for $\underline{\underline{\underline{D}}}^{k}$. 


\subsubsection{Performance}

Let us now evaluate the performances of the different methods proposed for the inversion of the state law of the model. We used the same test case as in Section 3.1.3 (see Figure 4), but the damage law considered was that of section 2.3.3, which is the most difficult behavior loop because damage increases until it reaches the criterion defined in (23) (see Figure 3). The methods are compared in terms of the number of iterations and time in Figure 6. Clearly, the secant operator performs very poorly and should not be used. The Aitken and BFGS operators have comparable performance in terms of both the number of iterations and time. Figure 6(b) shows that they lead to a gain by a factor 2 to 200 compared to the secant operator and reduce the cost of the inversion of the state law to approximately $50 \%$ of that of the local loop (see Figure 6(c)) even when damage approaches saturation. The BFGS operator, which is slightly more efficient than Aitken's, will be used from now on. In order to appreciate the importance of this gain, one should bear in mind that this loop is run at each local iteration of each global iteration of each time increment, which makes it the key factor in the performance of the implementation.

\section{Control of the damage localization}

The calculation of softening models such as those defined in Section 2.3.3 over a domain goes through a critical point where a loss of ellipticity, i.e. a loss of uniqueness of the solution [Needleman, 1988; Pijaudier-Cabot and Benallal, 1993; Peerlings et al., 1998], occurs and leads to pathological mesh dependencies [Ladevèze et al., 2000; Desmorat et al., 2010]. Several remedies exist for damage models, including nonlocal formulations [Pijaudier-Cabot 


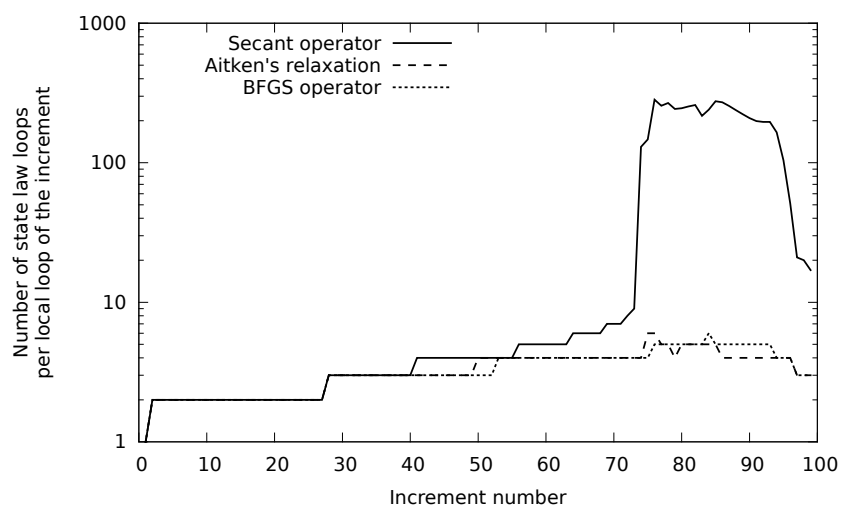

(a) iterations

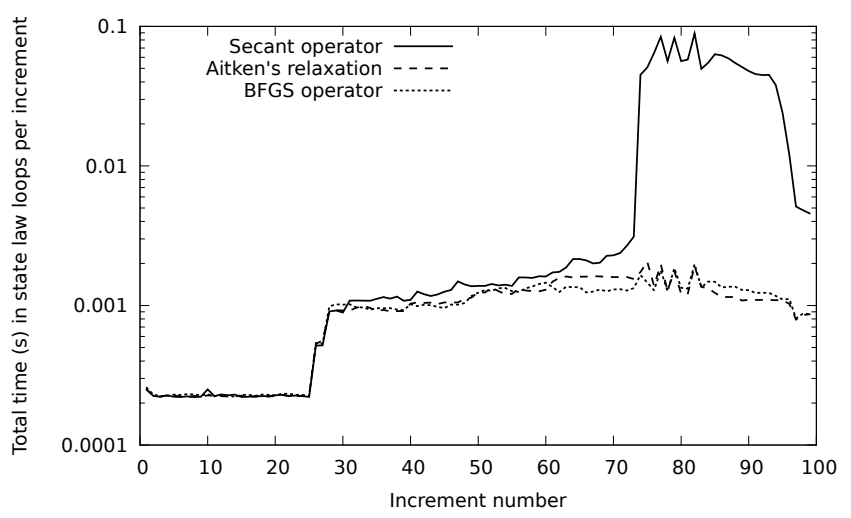

(b) time

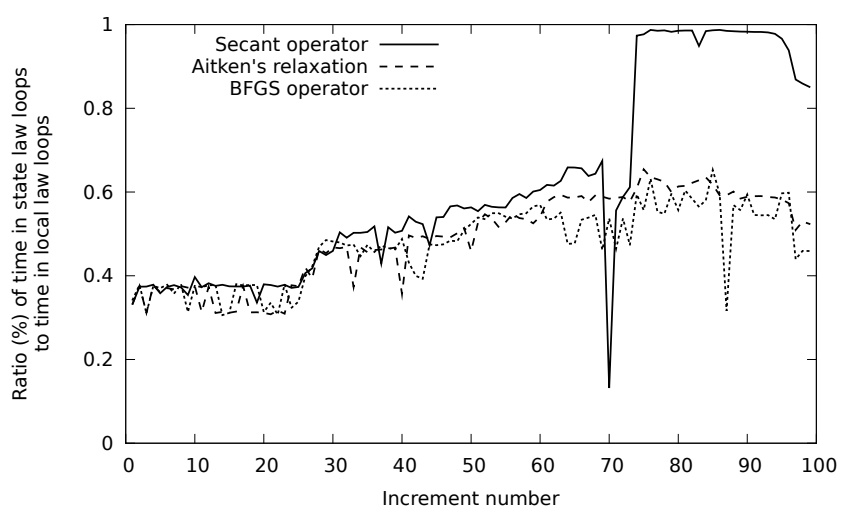

(c) time ratio

Figure 6: Performance of the state law inversion methods 
1 and Benallal, 1993; Peerlings et al., 1998; Voyiadjis et al., 2004; Desmorat

2 et al., 2010] and delay-effect approaches [Ladevèze et al., 2000; Kerfriden

3 et al., 2009; Desmorat et al., 2010]. The latter was chosen for our work

4 because it is a local method, which is easy to implement in FE codes such

5 as Abaqus/Standard.

6 4.1. The delay-effect method

$7 \quad$ First, in order to have a damage variable with an order of magnitude of 8 1, let us rewrite Equation (21) as follows:

$$
\left\{\begin{array}{ccc}
d & \text { if } & \sqrt{\bar{z}} \leq \epsilon_{0} \\
\left(\frac{\sqrt{\bar{z}}-\epsilon_{0}}{\epsilon_{1}-\epsilon_{0}}\right)^{2} & \text { if } & \epsilon_{0} \leq \sqrt{\bar{z}} \leq \epsilon_{1} \\
1 & \text { if } & \sqrt{\bar{z}} \geq \epsilon_{1}
\end{array}\right.
$$

9 Now, the delay-effect method for controlling the localization of damage con10 sists in replacing the evolution of this damage variable by an evolution with 11 a bounded rate:

$$
\dot{d}=\frac{1}{\tau_{c}}\left(1-e^{-\left\langle d^{\text {static }}-d\right\rangle_{+}}\right)
$$

12 where $d^{\text {static }}$ is the static function defined in Equation (32) and $\tau_{c}$ a ficti13 tious time parameter which must be chosen in order to avoid uncontrolled 14 localization [Ladevèze et al., 2000; Kerfriden et al., 2009]. The numerical 15 resolution of such a simple nonlinear, but scalar, equation is straightforward 16 and will not be discussed here.

17 The behavior law is modified with the introduction of this viscous-like 18 parameter. Figure 7 shows the stress-strain curves obtained with different 19 values of $\tau_{c}$. 


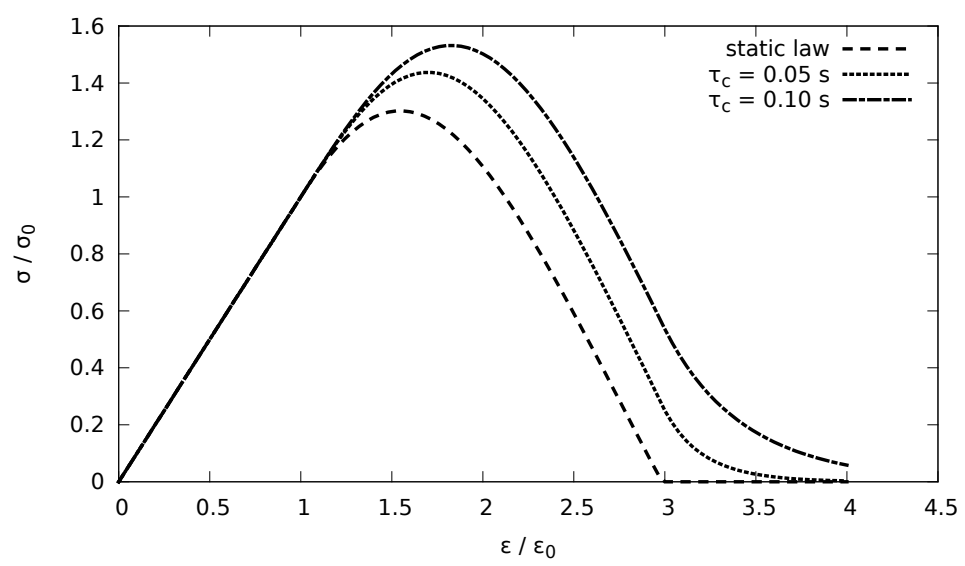

Figure 7: The response of the model under unidirectional tension loading $\left(k=10^{-5}\right.$, $\epsilon_{1} / \epsilon_{0}=3, \dot{\epsilon}_{1}^{g}=4 \epsilon_{0} / \mathrm{s}$ ) using different characteristic times for the delay-effect law

1 4.2. Illustrative examples

2 CT specimen. The effectiveness of the delay-effect method in controlling the 3 localization of damage was evaluated using a simple CT-like test, i.e. a pre4 cracked specimen subjected to tension (see Figure 8). The model consisted of 5 an initially isotropic material (with $E_{0}=250 \mathrm{MPa}$ and $\nu_{0}=0.3$ ) whose state 6 evolution was modeled using a microstructure-oriented softening damage 7 law (see Equations (19) and (20) of Section 2.3.3, with $a=0, n=2$ and $8 \underline{n}$ orthogonal to the initial crack) and the limited-rate kinetics of the delay9 effect method (see Equations (32) and (33) of Section 4.1, with $\epsilon_{0}=110^{-3}$, ${ }_{10} \epsilon_{1}=310^{-3}, k=10^{-5}$ and $\left.\tau_{c}=310^{-2} \mathrm{~s}\right)$. The response and the damage 11 fields for different meshes (generated with GMSH [Geuzaine and Remacle, 12 2009]) are shown in Figure 9. One can clearly observe that the solution is 13 objective, i.e. mesh-independent, in terms of both the mechanical response 14 and the damage fields. Indeed, while the response of the coarse mesh is 15 slightly different, the responses of the medium and fine meshes are very 
similar.

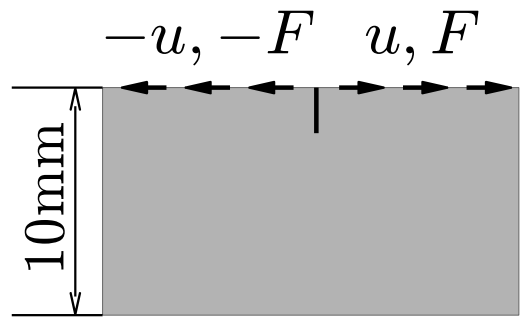

Figure 8: The problem used for the evaluation of localization control based on delay-effect damage ( $u$ is the applied displacement, $F$ is the calculated force, and $\dot{u}=0.2 \mathrm{~mm} / \mathrm{s}$ )

2 Plate with an open hole. Now let us consider a first structural example which 3 consists in a plate with an open hole (see Figure 10). The model was the

4 same as for the CT specimen, except for $\tau_{c}=110^{-2} \mathrm{~s}$. Figure 11 shows the 5 response of the model using different meshes (again generated with GMSH

6 [Geuzaine and Remacle, 2009]). Once again, the response of the coarse mesh 7 is slightly different, but the responses of the medium and fine meshes are 8 very similar, which attests to the fact that the method can be used to make 9 an objective prediction of the structure's final rupture.

\section{Conclusion}

11 This paper examined several key aspects of the formulation and numer12 ical implementation of a family of models belonging to the anisotropic and 13 unilateral damage theory. This modeling framework is based on a general 14 expression of the damaged elastic energy potential which enables one to 15 distinguish between tension behavior and compression behavior throughout 


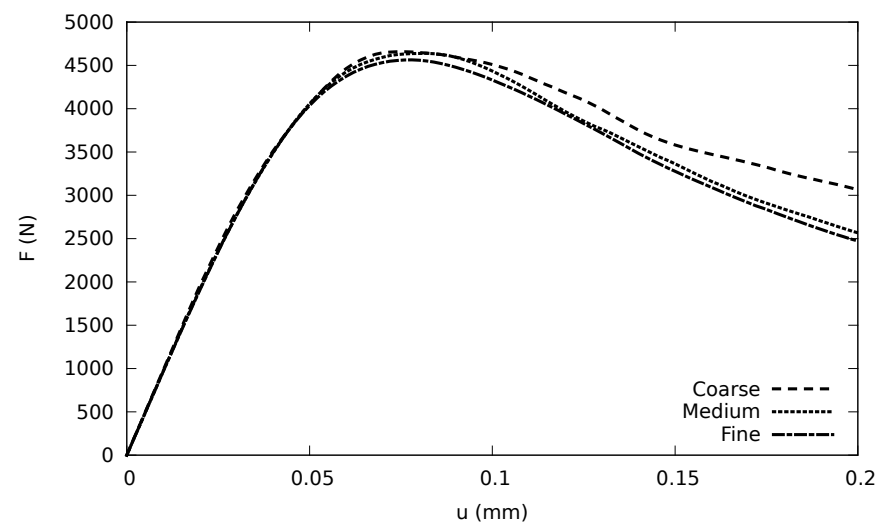

(a) Response

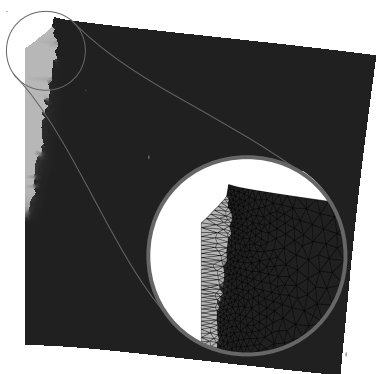

(b) Coarse mesh (2250 DOFs)

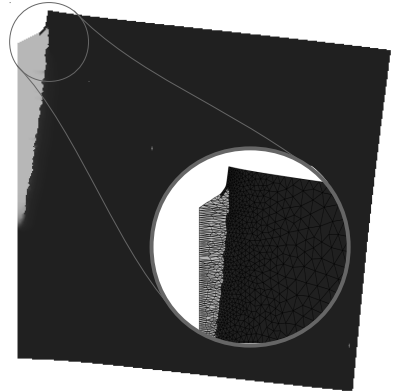

(c) Medium mesh $(3500$ DOFs)

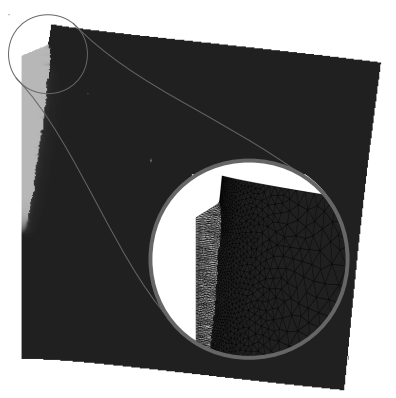

(d) Fine mesh (4550 DOFs)

Figure 9: The response and the damage field for the CT specimen at $t=0.75 \mathrm{~s}$ (dark grey: no damage; light gray: full damage criterion reached, see Equation (23) of Section 2.3.3): objectivity of the mesh and prediction of the crack's propagation 


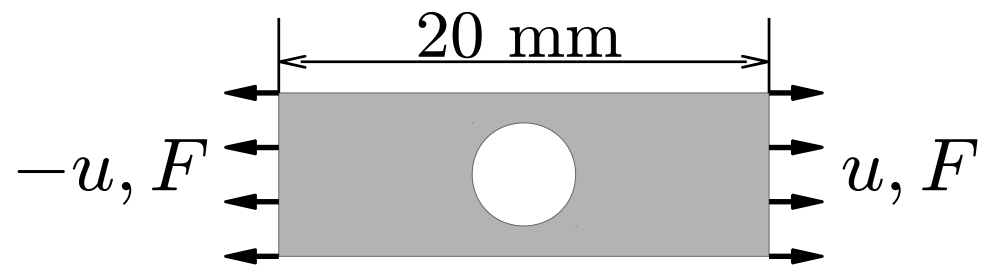

Figure 10: Structural case: plate with an open hole ( $u$ is the applied displacement, $F$ is the calculated force, and $\dot{u}=0.1 \mathrm{~mm} / \mathrm{s}$ )

1 the evolution of the damage, even when the orientation of the damage is not 2 known a priori (see Section 2.1).

3 Several damage evolution laws were presented in order to deal with all 4 types of damage: load-oriented or microstructure-oriented; reaching satura5 tion or localizing into a macroscopic crack (see Section 2.3). The formulation 6 of the microstructure-oriented damage law was modified slightly compared 7 to the previous formulation [Ladevèze, 2002] in order to make it equivalent 8 to the load-oriented laws when the load is normal to the crack (see Sec9 tion 2.3.2). In addition, softening laws were presented in this framework for the first time (see Section 2.3.3). Let us note that even though this paper 1 discusses only models with a single damage evolution law, it is possible to define models with several laws, each associated with a different damage mechanism. For example, in the model proposed for CMCs in [Ladevèze, 2002; Genet et al., 2012], three damage evolution laws were defined: one 15 load-oriented law for inter-yarn cracking and two microstructure-oriented laws for intra-yarn cracking of the longitudinal and transversal yarns.

Then we presented our current implementation of the whole family of models in Abaqus/Standard. The local equations are solved using a fixedpoint method. Among several relaxation schemes which were considered, 


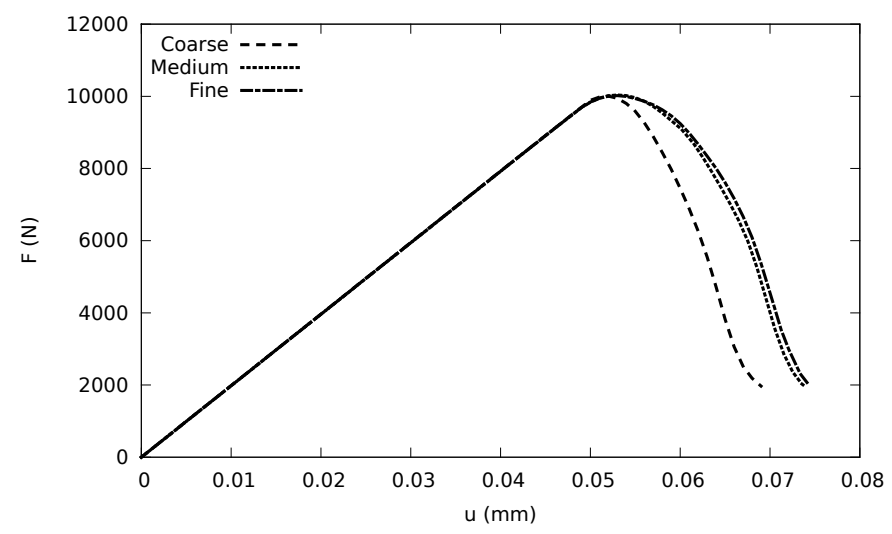

(a) Response
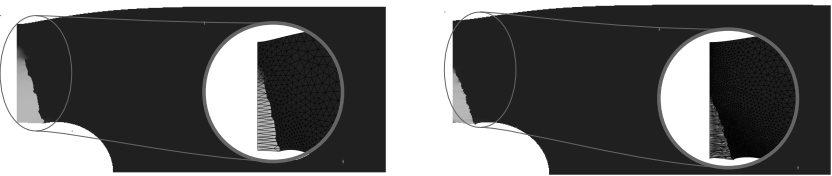

(b) Coarse mesh (1330

(c) Medium mesh $(5850$

DOFs)

DOFs)

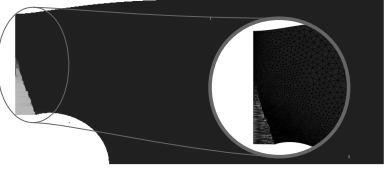

(d) Fine mesh (7100 DOFs)

Figure 11: The response and the damage field for the open hole specimen at $t=1 \mathrm{~s}$ (dark grey: undamaged; light gray: full damage criterion reached, see Equation (23) of Section 2.3.3): objectivity of the mesh and prediction of the final rupture 
1 Aitken's appears to be the most efficient and reduces the cost of the local 2 resolutions to approximately $10 \%$ of that of the global iterations (see Section 3 3.1). Since the model's state law is nonlinear even when all the internal 4 variables are fixed, its inversion is carried out using a Newton-Raphson 5 method. Once again, several acceleration schemes were considered, among 6 which the BFGS quasi-tangent method appears to be the most efficient. 7 This reduces the cost of inverting the state law to approximately $50 \%$ of 8 that of the local iterations, even with significant damage (see Section 3.2).

$9 \quad$ Finally, a delay-effect method was used to control damage localization 10 in the case of softening evolution laws. This enabled us to simulate the 11 propagation of a macroscopic crack and the final rupture of a structure 12 with no mesh dependency (see Section 4).

13 14 Regarding perspectives, since this framework has already been applied to 15 the case of CMCs [Ladevèze, 2002; Genet et al., 2012], the new capabilities 16 of simulating the creation and propagation of macroscopic cracks will replace 17 the simple criterion used until now (see [Cluzel et al., 2009; Genet et al., 18 2012]). In addition, microanalyses of cracked media will be carried out in 19 order to derive in a generic way some of the model parameters, such as those 20 which define the damage anisotropy (see Sections 2.3.1 and 2.3.2) or the 21 projectors (see Section 2.3.2). This has already been done for the kinematics 22 of a macroscopic damage model for concrete [Delaplace and Desmorat, 2007] 23 and for both the kinematics and the kinetics of a mesoscopic damage model 24 for laminated composites [Ladevèze and Lubineau, 2002]. 


\section{Acknowledgments}

This work was carried out as part of the joint research program "Modeling-Extrapolation-Validation of the lifetime of composites with self4 healing ceramic matrix" among Snecma Propulsion Solide, the DGA, the

5 CNRS, the CEAT, INSA Lyon, ENS Cachan, the University of Bordeaux 6 and the University of Perpignan; and part of the ARCOCE mechanical re7 search and technology program sponsored by Snecma Propulsion Solide and 8 the Région Aquitaine.

\section{References}

Xavier Aubard. Modelling of the mechanical behaviour of a 2-D SiC-SiC composite at a meso-scale. Composites Science and Technology, 54(95): 371-378, 1995.

Pierre Badel, Vincent Godard, and Jean-Baptiste Leblond. Application of some anisotropic damage model to the prediction of the failure of some complex industrial concrete structure. International Journal of Solids and Structures, 44(18-19):5848-5874, September 2007. ISSN 00207683. DOI 10.1016/j.ijsolstr.2007.02.001.

Ignacio Carol and Kaspar Willam. Spurious energy dissipation/generation in stiffness recovery models for elastic degradation and damage. International Journal of Solids and Structures, 33(20-22):2939-2957, August 1996. ISSN 00207683. DOI 10.1016/0020-7683(95)00254-5.

Jean-Louis Chaboche. Anisotropic creep damage in the framework of continuum damage mechanics. Nuclear Engineering and Design, 79(3):309-319, 1984 . 
Jean-Louis Chaboche. Continuum Damage Mechanics. 1. General concepts. Journal of Applied Mechanics - Transaction of the ASME, 55(1):59-64, March 1988.

Jean-Louis Chaboche. Damage induced anisotropy: on the difficulties associated with the active/passive unilateral condition. International Journal of Damage Mechanics, 1(2):148-171, April 1992. ISSN 1056-7895. DOI $10.1177 / 105678959200100201$.

Jean-Louis Chaboche. A continuum damage theory with anisotropic and unilateral damage. Recherche Aérospatiale, (2):139-147, 1995.

C. L. Chow and J. Wang. An anisotropic theory of continuum damage mechanics for ductile fracture. Engineering Fracture Mechanics, 27(5): 547-558, 1987a.

C. L. Chow and J. Wang. An anisotropic theory of elasticity for continuum damage mechanics. International Journal of Fracture, 33(1):3-16, January $1987 \mathrm{~b}$.

C. L. Chow and Y Wei. A model of continuum damage mechanics for fatigue failure. International Journal of Fracture, 50(4):301-316, August 1991.

Christophe Cluzel, Emmanuel Baranger, Pierre Ladevèze, and Anne Mouret. Mechanical behaviour and lifetime modelling of self-healing ceramicmatrix composites subjected to thermomechanical loading in air. Composites Part A: Applied Science and Manufacturing, 40(8):976-984, August 2009. ISSN 1359835X. DOI 10.1016/j.compositesa.2008.10.020.

J Cordebois and F Sidoroff. Anisotropic damage in elasticity and plasticity. Journal de Mécanique Théorique et Appliquée, pages 45-60, 1982. 
Arnaud Delaplace and Rodrigue Desmorat. Discrete 3D model as complimentary numerical testing for anisotropic damage. International Journal of Fracture, 148(2):115-128, November 2007. DOI 10.1007/s10704-0089183-9.

Rodrigue Desmorat. Quasi-unilateral conditions in anisotropic elasticity. Comptes Rendus à l'Académie des Sciences, Série IIB-Mécanique, 328 (6):445-450, June 2000.

Rodrigue Desmorat, Fabrice Gatuingt, and Frédéric Ragueneau. Nonlocal anisotropic damage model and related computational aspects for quasibrittle materials. Engineering Fracture Mechanics, 74(10):1539-1560, July 2007. ISSN 00137944. DOI 10.1016/j.engfracmech.2006.09.012.

Rodrigue Desmorat, Marion Chambart, Fabrice Gatuingt, and D Guilbaud. Delay-active damage versus non-local enhancement for anisotropic damage dynamics computations with alternated loading. Engineering Fracture Mechanics, 77(12):2294-2315, August 2010. DOI 10.1016/j.engfracmech.2010.04.006.

Martin Genet, Lionel Marcin, Emmanuel Baranger, Christophe Cluzel, Pierre Ladevèze, and Anne Mouret. Computational prediction of the lifetime of self-healing CMC structures. Composites Part A: Applied Science and Manufacturing, 43(2):294-303, February 2012. ISSN 1359835X. DOI 10.1016/j.compositesa.2011.11.004.

Christophe Geuzaine and Jean-François Remacle. Gmsh: a threedimensional finite element mesh generator with built-in pre- and postprocessing facilities. International Journal for Numerical Methods in 
Engineering, 79(11):1309-1331, September 2009. ISSN 00295981. DOI 10.1002/nme.2579.

L. Gorbatikh, D. S Ivanov, S. V. Lomov, and Ignace Verpoest. On modelling of damage evolution in textile composites on meso-level via property degradation approach. Composites Part A: Applied Science and Manufacturing, 38(12):2433-2442, December 2007. ISSN 1359835X. DOI 10.1016/j.compositesa.2007.08.017.

L. M. Kachanov. Rupture time under creep conditions (in Russian). Izvestia Akademii Nauk SSSR, Otdelenie tekhnicheskich nauk, (8):26-31, 1958.

L. M. Kachanov. Introduction to Continuum Damage Mechanics. Mechanics of Elastic Stability. H.H.E. Leipholz and G.AE. Oravas, 1986.

L. M. Kachanov. Rupture time under creep conditions. International Journal of Fracture, (98):6-13, 1999.

Christophe Kassiotis, Adnan Ibrahimbegovic, and Hermann Matthies. Partitioned solution to fluid-structure interaction problem in application to free-surface flows. European Journal of Mechanics - B/Fluids, 29(6):510521, 2010. DOI DOI: 10.1016/j.euromechflu.2010.07.003.

Pierre Kerfriden, Olivier Allix, and Pierre Gosselet. A three-scale domain decomposition method for the 3D analysis of debonding in laminates. Computational Mechanics, 44(3):343-362, August 2009. DOI 10.1007/s00466009-0378-3.

D. Krajcinovic. Damage Mechanics, volume 8 of Applied Mathematics and Mechanics. North-Holland, December 1996. 
Pierre Ladevèze. On an anisotropic damage Theory (in French). Internal report 34, LMT-Cachan, March 1983.

Pierre Ladevèze. Modeling and Simulation of the Mechanical Behavior of CMCs. High-Temperature Ceramic-Matrix Composites, 47:53-63, 1995.

Pierre Ladevèze. Nonlinear Computational Structural Mechanics: New Approaches and Non-Incremental Methods of Calculation. Mechanical Engineering. Springer, 1999.

Pierre Ladevèze. An anisotropic damage theory with unilateral effects: applications to laminate and three- and four-dimensional composites. In Olivier Allix and François Hild, editors, Continuum Damage Mechanics of Materials and Structures, pages 205-233. Elsevier, 2002.

Pierre Ladevèze and Stéphane Letombe. Recent advances on an anisotropic damage theory including unilateral effects. In Proceedings of the International Bimestre on Damage Mechanics, Symposium on Continuous Damage and Fracture, 2000.

Pierre Ladevèze and Gilles Lubineau. An enhanced mesomodel for laminates based on micromechanics. Composites Science and Technology, 62:533$541,2002$.

Pierre Ladevèze, Alain Gasser, and Olivier Allix. Damage mechanisms modelling for ceramic composites. Journal of Engineering Materials and Technology, 116:331-336, 1994.

Pierre Ladevèze, Olivier Allix, Jean-François Deü, and David Lévêque. A mesomodel for localisation and damage computation in laminates. Computer Methods in Applied Mechanics and Engineering, 183:105-122, 2000. 
Marie-Christine Lafarie-Frenot, C. Hénaff-Gardin, and D. Gamby. Matrix cracking induced by cyclic ply stresses in composite laminates. Composites Science and Technology, 61(15):2327-2336, November 2001. ISSN 02663538. DOI 10.1016/S0266-3538(01)00125-7.

Jacques Lamon. A micromechanics-based approach to the mechanical behavior of brittle-matrix composites. Composites Science and Technology, 61(15):2259-2272, November 2001. ISSN 02663538. DOI 10.1016/S02663538(01)00120-8. URL www. scopus.com.

Frederick A. Leckie. Constitutive equations of continuum creep damage mechanics. Philosophical Transactions of the Royal Society of London Series A-Mathematical Physical and Engineering Sciences, 288(1350):2747, 1978.

Hugo Leclerc. Towards a no compromise approach between modularity, versatility and execution speed for computational mechanics on CPUs and GPUs. In IV European Conference on Computational Mechanics (ECCM2010), 2010.

Jean Lemaître. A continuous damage mechanics model for ductile fracture. Journal of Engineering Materials and Technology - Transactions of the ASME, 107(1):83-89, 1985.

Jean Lemaître. A Course on Damage Mechanics. Springer-Verlag, 1992.

Jean Lemaître and Rodrigue Desmorat. Engineering Damage Mechanics: Ductile, Creep, Fatigue and Brittle Failures. Springer, 2005.

Jean Lemaître, Jean-Louis Chaboche, Rodrigue Desmorat, and Ahmed Be- 
nallal. Solid Materials Mechanics, Third Edition (In French). Dunod, 2009.

P. M. Lesne and K. Saanouni. Modeling of irreversible damage-induced strains in brittle elastic composites. Recherche Aérospatiale, (2):23-37, 1993. ISSN 0034-1223.

Hermann Matthies and G Strang. Solution of non-linear finite-element equations. International Journal for Numerical Methods in Engineering, 14 (11):1613-1626, 1979.

S. Murakami. Notion of continuum damage mechanics and its application to anisotropic creep damage theory. Journal of Engineering Materials and Technology - Transactions of the ASME, 105(2):99-105, 1983.

Alan Needleman. Material rate dependance and mesh sensitivity in localization problems. Computer Methods in Applied Mechanics and Engineering, 67(1):69-85, March 1988.

Jean-Charles Passieux, Pierre Ladevèze, and David Néron. A scalable timespace multiscale domain decomposition method: adaptive time scale separation. Computational Mechanics, 46(4):621-633, September 2010. DOI 10.1007/s00466-010-0504-2.

R.H.J. Peerlings, René De Borst, W A M Brekelmans, and M.G.D. Geers. Gradient-enhanced damage modelling of concrete fracture. Mechanics of Cohesive-Frictional Materials, 3(4):323-342, October 1998.

Vincent Pensée, Djimédo Kondo, and Luc Dormieux. Micromechanical analysis of anisotropic damage in brittle materials. Journal of Engineer- 
ing Mechanics, 128(8):889-897, August 2002. DOI 10.1061/(ASCE)07339399(2002)128:8(889).

Gilles Pijaudier-Cabot and Ahmed Benallal. Strain localization and bifurcation in a nonlocal continuum. International Journal of Solids and Structures, 30(13):1761-1775, 1993.

J.C. Simo and J.W. Ju. Strain-based and stress-based continuum damage models. 1. Formulation. International Journal of Solids and Structures, 23(7):821-840, 1987.

Ramesh Talreja. Transverse Cracking and Stiffness Reduction in Composite Laminates. Journal of Composite Materials, 19(4):355-375, January 1985. ISSN 0021-9983. DOI 10.1177/002199838501900404.

Ramesh Talreja. Stiffness properties of composite laminates with matrix cracking and interior delamination. Engineering Fracture Mechanics, 25(5-6):751-762, January 1986. ISSN 00137944. DOI 10.1016/00137944(86)90038-X.

George Z. Voyiadjis and P I Kattan. Damage of fiber-reinforced compositematerials with micromechanical characterization. International Journal of Solids and Structures, 30(20):2757-2778, 1993.

George Z. Voyiadjis, Rashid K. Abu Al-Rub, and Anthony N. Palazotto. Thermodynamic framework for coupling of non-local viscoplasticity and non-local anisotropic viscodamage for dynamic localization problems using gradient theory. International Journal of Plasticity, 20(6):981-1038, June 2004. ISSN 07496419. DOI 10.1016/j.ijplas.2003.10.002. 